\title{
Exact Evaluation of Stochastic Watersheds: From Trees to General Graphs
}

\author{
Filip Malmberg, Bettina Selig, and Cris L. Luengo Hendriks \\ Centre for Image Analysis, \\ Uppsala University and Swedish University of Agricultural Sciences, Sweden \\ ffilip, bettina, cris\}@cb.uu.se
}

\begin{abstract}
The stochastic watershed is a method for identifying salient contours in an image, with applications to image segmentation. The method computes a probability density function (PDF), assigning to each piece of contour in the image the probability to appear as a segmentation boundary in seeded watershed segmentation with randomly selected seedpoints. Contours that appear with high probability are assumed to be more important. This paper concerns an efficient method for computing the stochastic watershed PDF exactly, without performing any actual seeded watershed computations. A method for exact evaluation of stochastic watersheds was proposed by Meyer and Stawiaski (2010). Their method does not operate directly on the image, but on a compact tree representation where each edge in the tree corresponds to a watershed partition of the image elements. The output of the exact evaluation algorithm is thus a PDF defined over the edges of the tree. While the compact tree representation is useful in its own right, it is in many cases desirable to convert the results from this abstract representation back to the image, e.g, for further processing. Here, we present an efficient linear time algorithm for performing this conversion.
\end{abstract}

Keywords: Stochastic watershed, Watershed cut, Minimum spanning tree.

\section{Introduction}

The stochastic watershed, proposed by Angulo and Jeulin 1, is a method for identifying salient contours in an image, with applications to image segmentation. This method is based on the seeded watershed [14, which partitions the image into regions according to a set of seedpoints, so that every region contains precisely one seed and the boundaries between regions are optimally aligned with strong gradients in the image [6]. The stochastic watershed estimates the strength of edges in the image by repeatedly performing seeded watershed segmentation with randomly selected seedpoints. Each repetition will find a different subset of edges, but more important edges will be found more frequently. The output of the method is a probability density function (PDF), assigning to each piece of contour in the image the probability to appear as a segmentation boundary in seeded watershed segmentation with $N$ randomly selected seedpoints. 
Here, we study watersheds on edge weighted graphs. In this context, a digital image is commonly represented by its pixel adjacency graph, i.e., a graph where each image element corresponds to a vertex in the graph, and adjacent image elements are connected by graph edges. Consequently, the seedpoints used for watershed segmentation consist of a set of vertices in the graph, and the watershed itself is defined as a watershed cut [67]. Informally, a cut is a set of edges that, if removed from the graph, separates it into two or more connected components. The stochastic watershed PDF is then defined as a mapping over the edges of the graph, i.e., every edge is considered to be a "piece of contour".

In the original paper by Angulo and Jeulin [1], the PDF was estimated by Monte Carlo simulation, i.e., repeatedly selecting $N$ random seedpoints and performing seeded watershed segmentation. The drawback of this approach is that a large number of watershed segmentations must be performed to obtain a good estimate of the PDF. Meyer and Stawiaski [15] showed that the PDF can be calculated exactly, without performing any Monte Carlo simulations. Their work was later extended by Malmberg and Luengo [13], who proposed an efficient (pseudo-linear) algorithm for computing the exact PDF.

The exact evaluation method does not operate directly on the pixel adjacency graph $G$, but on a minimum spanning tree of $G$. The minimum spanning tree provides a compact representation of all watershed cuts on $G$ in the sense that, under certain conditions, there is a one-to-one correspondence between the watershed cuts on $G$ and the watershed cuts on its minimum spanning tree. The output of the exact evaluation algorithm is thus a map from the edges of the minimum spanning tree to $[0,1]$ such that the value of the map for a given edge equals the probability of that edge being included in the watershed cut on the tree for a set of randomly selected seedpoints.

The compact tree representation is useful in its own right. For example, it is straightforward to obtain a segmentation directly from the tree by removing a set of edges with high probability values, and performing connected component labeling on the resulting forest. In general, the tree representation can be treated as any other segmentation hierarchy for morphological segmentation 8 . Nevertheless, there are cases where it might be useful to extend the stochastic watershed PDF to all edges in the graph. Malmberg and Luengo [13] suggested that such an extension could be performed using the saliency map approach of Najman and Schmitt [17/9. As will be shown, however, this does not lead to the correct PDF for stochastic watersheds. Here, we propose an efficient algorithm for performing the correct extension. We show that for sparse graphs, common in image analysis applications, the time complexity of the proposed algorithm is $\mathcal{O}(|V|)$, where $|V|$ is the number of vertices in the graph. 


\section{Preliminaries}

\subsection{Edge Weighted Graphs}

We will formulate our results in the framework of edge weighted graphs. In this context, a digital image is represented by its pixel adjacency graph, where each image element corresponds to a vertex in the graph, and adjacent image elements are connected by graph edges. In this section, we introduce some basic definitions to handle edge weighted graphs.

We define a graph as a triple $G=(V, E, \lambda)$ where

$-V$ is a finite set.

- $E$ is a set of unordered pairs of distinct elements in $V$, i.e., $E \subseteq\{\{v, w\} \subseteq V$ $\mid v \neq w\}$.

$-\lambda$ is a map $\lambda: E \rightarrow \mathbb{R}$.

The elements of $V$ are called vertices of $G$, and the elements of $E$ are called edges of $G$. When necessary, $V, E$, and $\lambda$ will be denoted $V(G), E(G)$, and $\lambda(G)$ to explicitly indicate which graph they belong to. An edge spanning two vertices $v$ and $w$ is denoted $e_{v, w}$. If $e_{v, w}$ is an edge in $E$, the vertices $v$ and $w$ are adjacent. The neighborhood of a vertex $v$ it the set of all vertices adjacent to $v$, and is denoted by $\mathcal{N}(v)$.

For any edge $e \in E, \lambda(e)$ is the weight or altitude of $e$. Throughout the paper, we will assume that the value of $\lambda(e)$ represents the dissimilarity between the vertices spanned by $e$. Thus, we assume that the salient contours are located on the highest edges of the graph. In the context of image processing, we may define the edge weights as, e.g.,

$$
\lambda\left(e_{v, w}\right)=|I(v)-I(w)|,
$$

where $I(v)$ and $I(w)$ are the intensities of the image elements corresponding to the vertices $v$ and $w$, respectively.

The seeded watershed method, and hence also the method presented here, depends on an increasing order of the edge weights in a graph, but not on their exact value [6:7. To ensure the uniqueness of the seeded watershed segmentation, we will only consider graphs where each edge has a unique weight, thereby ensuring a unique increasing order. Graphs that do not fulfill this property can be easily be converted to the correct format as follows:

1. Fix an increasing ordering of the graph edges, i.e., find a map $O: E \rightarrow \mathbb{Z}$ such that $e_{i} \neq e_{j} \Rightarrow O\left(e_{i}\right) \neq O\left(e_{j}\right)$ and $O\left(e_{i}\right)<O\left(e_{j}\right) \Rightarrow \lambda\left(e_{i}\right) \leq \lambda\left(e_{j}\right)$ for all $e_{i}, e_{j} \in E$.

2. For all $e \in E$, set $\lambda(e) \leftarrow O(e)$. 
Let $G$ be a graph. A path in $G$ is an ordered sequence of vertices $\pi=\left\langle v_{i}\right\rangle_{i=1}^{k}=$ $\left\langle v_{1}, v_{2}, \ldots, v_{k}\right\rangle$ such that $e_{v_{i}, v_{i+1}} \in E$ for all $i \in[1, k-1]$. We denote the origin $v_{1}$ and the destination $v_{k}$ of $\pi$ by $\operatorname{org}(\pi)$ and $\operatorname{dst}(\pi)$, respectively. The set of vertices $\left\{v_{1}, v_{2}, \ldots, v_{k}\right\}$ along $\pi$ is denoted $V(\pi)$, and the set of edges $\left\{e_{v_{i}, v_{i+1}} \mid i \in\right.$ $[1, k-1]\}$ along $\pi$ is denoted $E(\pi)$. A path that has no repeated vertices is said to be simple. Two vertices $v$ and $w$ are linked in $G$ if there exists a path $\pi$ in $G$ such that $\operatorname{org}(\pi)=v$ and $\operatorname{dst}(\pi)=w$. The notation $v \underset{G}{\sim} w$ will here be used to indicate that $v$ and $w$ are linked on $G$. If all pairs of vertices in $G$ are linked, then $G$ is connected, otherwise it is disconnected.

Let $G$ and $H$ be two graphs. If $V(H) \subseteq V(G)$ and $E(H) \subseteq E(G)$, then $H$ is a subgraph of $G$. If $H$ is a connected subgraph of $G$ and $v \neq w$ for all vertices $v \in H$ and $w \notin H$, then $H$ is a connected component of $G$.

Let $G$ be a graph and let $\pi$ be a path in $G$. If $\operatorname{dst}(\pi)=\operatorname{org}(\pi)$, then $\pi$ is a cycle. A cycle is simple if it has no repeated vertices other than the endpoints. If $G$ has no simple cycles, then $G$ is a forest. A connected forest is called a tree.

Let $G$ be a graph, and let $T$ be a subgraph of $G$ such that $T$ is a tree and $V(G)=V(T)$. Then $T$ is a spanning tree for $G$. The weight of a tree is the sum of all edge weights in the tree. A minimum spanning tree of $G$ is a spanning tree with weight less than or equal to the weight of every other spanning tree of $G$.

\subsection{Exact Stochastic Watersheds}

In this section, we briefly review the method of Meyer and Stawiaski [15] for exact evaluation of stochastic watersheds.

Let $G$ be a graph. In the method of Meyer and Stawiaski, the hierarchy of watershed segmentations is represented by a flooding tree, $T$, which in our context is equivalent to a minimum spanning tree $T$ of the graph $G$. Since $T$ has no simple cycles, every set of edges $S \subseteq E(T)$ forms a cut on $T$. Moreover, every set of edges $S \subseteq E(T)$ corresponds to a cut $S^{\prime}$ in $G$, given by

$$
S^{\prime}=\left\{e_{v, w} \in E(G) \mid \underset{(V(T), E(T) \backslash S)}{v \underset{w}{\sim}}\right\}
$$

We say that $S^{\prime}$ is the cut on $G$ induced by $S$. If $S$ is a watershed cut on $T$ with respect to some set of seedpoints, then the cut induced by $S$ is a watershed cut on $G$ with respect to the same seedpoints [6]7. In fact, there is a one-toone correspondence between the set of all watershed cuts on $T$ and the set of all watershed cuts on $G$. Meyer and Stawiaski [15] used this correspondence to compactly represent the stochastic watershed PDF as a mapping $P: E(T) \rightarrow$ $[0,1]$, where $P(e)$ is the probability of $e$ being included in the watershed cut on $T$ for $N$ randomly selected seedpoints. This mapping can be computed in $\mathcal{O}(|E| \alpha(|V|))$ time using the algorithm proposed by Malmberg and Luengo [13], where $\alpha$ is the extremely slow-growing inverse of the Ackermann function [5]. 


\section{Method}

Let $G$ be a graph, and let $T$ be a minimum spanning tree for $G$. For all $e \in E(T)$, let $P(e)$ be the probability of $e$ being included in the watershed cut on $T$ for $N$ randomly selected seedpoints. We will now show how the mapping $P$ defined over the edges of $T$ can be used to compute a mapping $P^{\prime}: E(G) \rightarrow[0,1]$ such that $P^{\prime}(e)$ is equal to the probability of $e$ being included in the watershed cut on $G$ for $N$ randomly selected seedpoints.

Malmberg and Luengo 13 suggested that the mapping $P$ could be extended to all edges in $E(G)$ using the saliency map approach of Najman and Schmitt [17] For every edge $e_{v, w} \in E(G)$, there is a unique path, denoted $\pi_{v, w}$, on $T$ connecting the vertices spanned by the edge. According to the saliency map approach, the mapping $P^{\prime}$ would be defined as

$$
P^{\prime}\left(e_{v, w}\right)=\max _{e \in E\left(\pi_{v, w}\right)}(P(e)) .
$$

By this definition, however, $P^{\prime}(e)$ does not equal the probability of $e$ being included in the watershed cut on $G$ for $N$ randomly selected seedpoints, as shown below.

Theorem 1. Let $S$ be a cut on $T$, and let $S^{\prime}$ be the cut on $G$ induced by $S$. For every edge $e_{v, w} \in E(G)$, it holds that $e_{v, w} \in S^{\prime}$ if and only if $S \cap E\left(\pi_{v, w}\right) \neq \emptyset$.

Proof. If $E\left(\pi_{v, w}\right) \cap S=\emptyset$, then $\pi_{v, w}$ is a path between $v$ and $w$ on $(V, E(T) \backslash S)$, i.e., $\underset{((V, E(T) \backslash S))}{v \sim w}$, and so $e_{v, w} \notin S^{\prime}$. Conversely, if $E\left(\pi_{v, w}\right) \cap S \neq \emptyset$, then $\underset{((V, E(T) \backslash S))}{v \not w}$, and so $e_{v, w} \in S^{\prime}$.

The probability of $P^{\prime}\left(e_{v, w}\right)$ of $e_{v, w}$ being included in the watershed cut on $G$ is therefore equal to the probability that the watershed cut on $T$ contains at least one edge along the path $\pi_{v, w}$ connecting $v$ and $w$ on $T$. This probability is given by

$$
P^{\prime}\left(e_{v, w}\right)=1-\prod_{e \in E\left(\pi_{v, w}\right)}(1-P(e)) .
$$

The derivation of Equation 4 is straightforward. Since the probability of an edge along $\pi_{v, w}$ being part of the watershed cut on $T$ is independent of that for other edges along $\pi_{v, w}$, the probability that no edge in $E\left(\pi_{v, w}\right)$ belongs to the watershed cut can be computed by multiplication of the individual probabilities. Note that for all edges $e_{v, w} \in E(T)$, Equation 4 reduces to $P^{\prime}\left(e_{v, w}\right)=P\left(e_{v, w}\right)$ as expected.

Based on the above results, we can formulate a naive approach for calculating $P^{\prime}\left(e_{v, w}\right)$ as follows: 


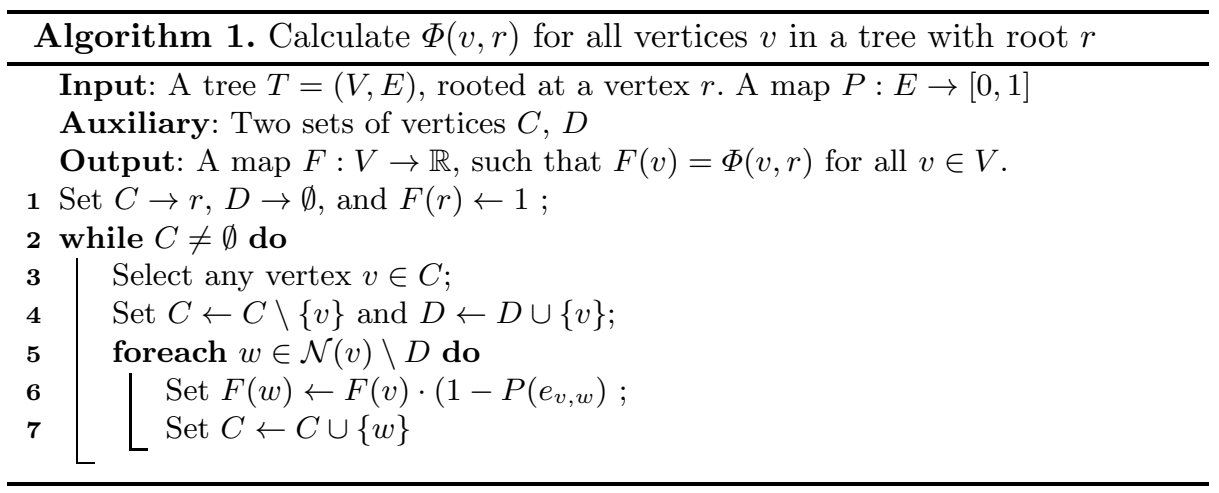

1. Find the unique path $\pi_{v, w}$ connecting $v$ and $w$ on $T$ using, e.g., breadth-first search.

2. Use Equation 4 to calculate $P^{\prime}\left(e_{v, w}\right)$.

Performing these calculations for all edges in a graph, however, is prohibitively slow. We will now present an efficient algorithm for computing $P^{\prime}(e)$ for all $e \in E(G)$. First, we define the function $\Phi(a, b)$ as

$$
\Phi(a, b)=\prod_{e \in E\left(\pi_{a, b}\right)}(1-P(e))
$$

Next, we designate an arbitrary vertex $r \in V$ to be the root of $T$. The choice of $r$ does not affect the output of the algorithm. For a pair of vertices $v, v^{\prime} \in V$, we say that $v$ is an ancestor of $v^{\prime}$ if $v$ lies along the path from $v^{\prime}$ to $r$ on $T$. The lowest common ancestor $\operatorname{LCA}(v, w)$ of two vertices $v$ and $w$ is the vertex located farthest from the root that is an ancestor of both $v$ and $w$. With these definitions in place, we can rewrite $\Phi(v, w)$ as

$$
\Phi(v, w)=\frac{\Phi(v, r) \Phi(w, r)}{\Phi(\operatorname{LCA}(v, w), r)^{2}} .
$$

For a fixed root $r$, the value of $\Phi(v, r)$ for all vertices $v \in V$ can be computed using Algorithm 1. Computationally, this algorithm is equivalent to breadth-first search, and so has the same $\mathcal{O}(|V|+|E(T)|)=\mathcal{O}(|V|)$ time complexity.

Once the value of $\Phi(v, r)$ has been calculated for all $v \in V$, Algorithm 2 can be used to calculate the desired probability $P^{\prime}(e)$ for all $e \in E(G)$. Algorithm 2 iterates over all edges of $G$, and computes the LCA of the vertices spanned by the edge. The time complexity of Algorithm 2 is therefore $\mathcal{O}(|E| \cdot X)$, where $X$ is the cost of finding the LCA between a pair of vertices. As shown by Harel and Tarjan [12, the LCA of a pair of vertices can be found in $\mathcal{O}(1)$ time, after performing an $\mathcal{O}(|V|)$ preprocessing step. An algorithm satisfying these bounds, while also being suitable for practical implementation, was proposed by Bender and Farach-Colton 3]. 


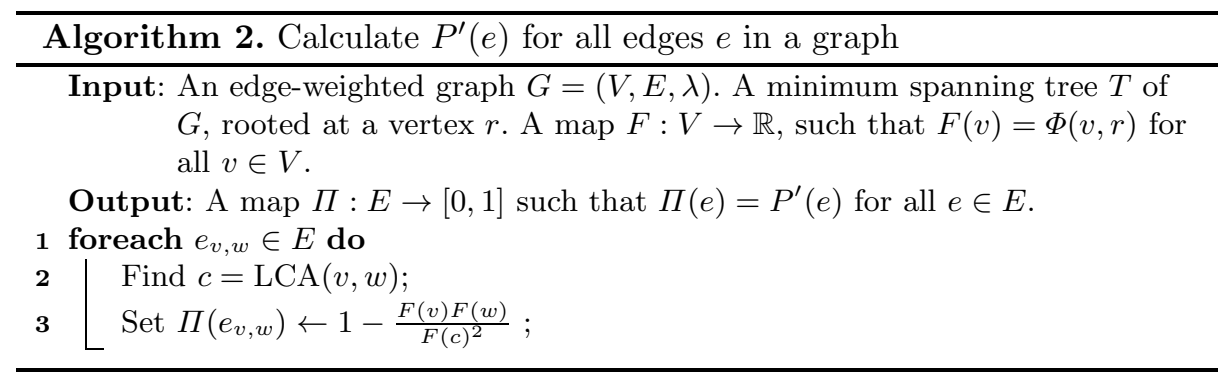

In total, the time complexity of the proposed method for calculating $P^{\prime}$ given $P$ is therefore $\mathcal{O}(|V|+|E(G)|)$. For the sparse graphs typically encountered in image processing it holds that $\mathcal{O}(|E(G)|)=\mathcal{O}(|V|)$, and for such graphs the total time complexity of the proposed method is therefore $\mathcal{O}(|V|)$.

\section{Visualizing the Probability Density Function}

In the common case where $G$ is a pixel adjacency graph, i.e., the vertices of $G$ correspond to image elements, it may be of interest to visualize the stochastic watershed PDF on the vertices of the graph. To this end, we introduce the notion of a boundary operator. A boundary operator $\delta$ is a mapping $\delta: V \rightarrow \mathcal{P}(V)$, such that $\delta(v) \subseteq \mathcal{N}(v)$ for all $v \in V$. Given a cut $S$ on $G$ and a boundary operator $\delta$, we say that a vertex $v$ is a boundary vertex for $S$ and $\delta$ if any of the vertices in $\delta(v)$ are separated from $v$ by the cut $S$. The probability $P^{\prime \prime}(v)$ of $v$ being a boundary vertex is then given by

$$
P^{\prime \prime}(v)=1-\prod_{w \in \delta(v)}\left(1-P^{\prime}\left(e_{v, w}\right)\right) .
$$

The derivation of Equation 7 is analogous to the derivation of Equation 4.

\section{Experiments}

As shown in Section 3, the asymptotic time required for computing the stochastic watershed PDF on the edges of the MST of a graph is the same as that required for computing the PDF on all edges of the graph. In this section, we investigate how much the the computation increases in practice when the PDF is computed for all edges, rather than on the edges of the MST only.

We calculate stochastic watershed PDFs on a set of two-dimensional images of varying sizes, generated by scaling up an original low resolution image using bi-cubic interpolation. Pixel adjacency graphs were constructed from the images using a standard 4-connected adjacency relation with edge weights defined according to Equation 1, For every pixel $\mathbf{x}$ in an image, the boundary operator 


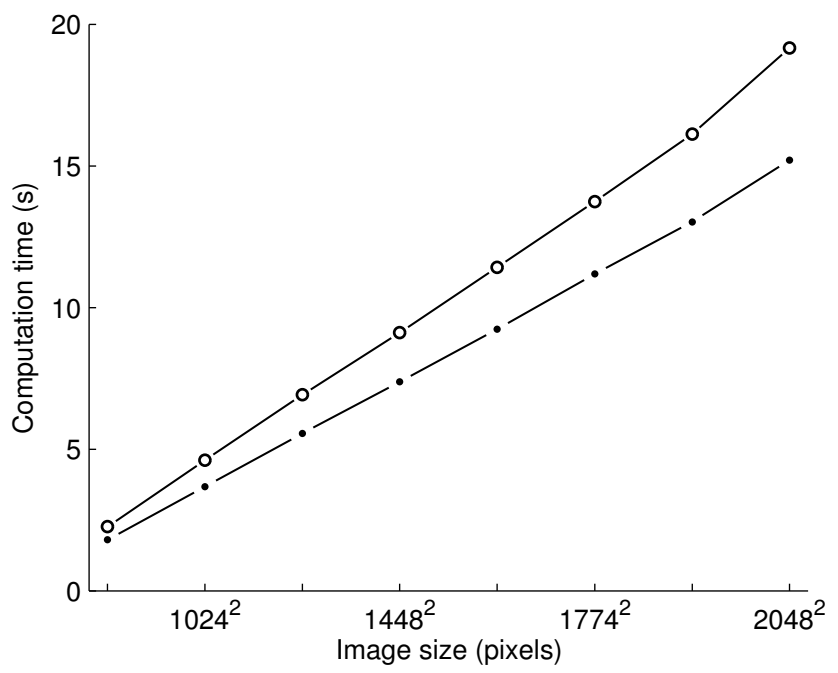

Fig. 1. Computation time for calculating the exact stochastic watershed PDF on pixel adjacency graphs for 2D images of varying sizes. The bottom curve shows the computation time for calculating the exact stochastic watershed PDF on the MST of the pixel adjacency graph using the method proposed by Malmberg and Luengo 13. The top curve shows the total computation time for calculating the stochastic watershed PDF for all edges in the pixel adjacency graph, using the proposed method to extend the PDF from the edges of the MST to all edges in the graph.

$\delta(\mathbf{x})$ was defined as $\delta(\mathbf{x})=\{\mathbf{x}+(1,0), \mathbf{x}+(0,1)\}$. For all pixel adjacency graphs, the exact stochastic watershed was first calculated on the MST of the pixel adjacency graph using the method of Malmberg and Luengo [13, and the PDF was then extended to all edges in the graph using the proposed method. For all experiments, $\mathrm{N}=20$ random seedpoints were used. Figure 1 shows the total computation time for both these steps, as well as the computation time for the first step only. As the figure shows, the overhead for extending the PDF to all edges is small - the average increase in computation time is about $25 \%$. Figure 2 shows the stochastic watershed PDF computed on the original low resolution 2D image. For reference, the same PDF approximated by Monte Carlo simulation with 1000 repetitions is also shown. At each repetition, we calculate the watershed cut corresponding to a random set of seedpoints and identify the boundary pixels using the boundary operator defined above. The final PDF is obtained by counting the number of times each pixel appears as a boundary pixel, and dividing this number by the total number of repetitions. 

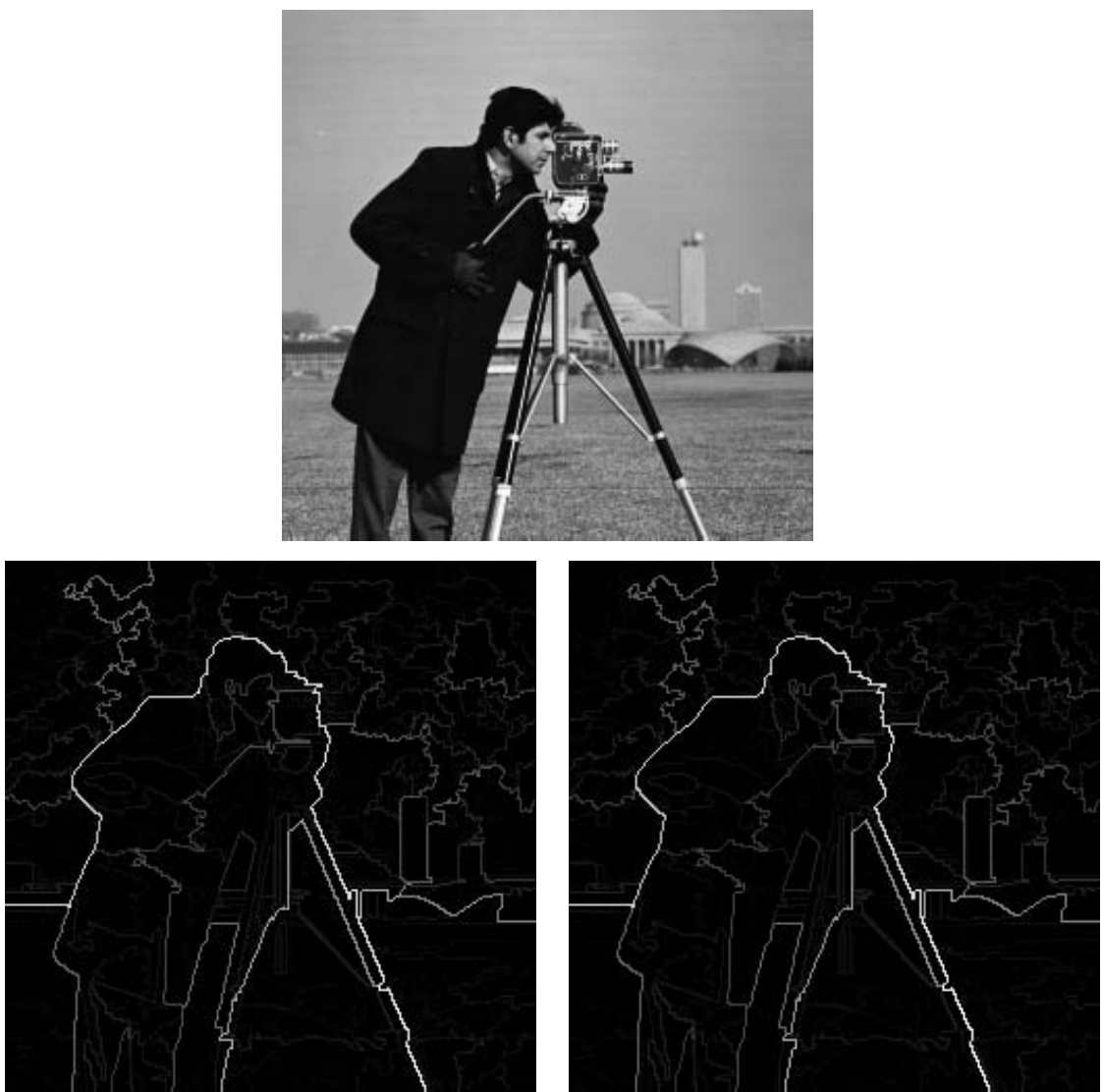

Fig. 2. The stochastic watershed PDF computed on a $2 \mathrm{D}$ image. The contrast of the images has been adjusted for display purposes. (Top) Original image. (Bottom left) PDF obtained by the proposed exact method. (Bottom right) Reference PDF obtained by Monte Carlo simulation with 1000 repetitions. 


\section{Conclusions}

The stochastic watershed method has found some applications, such as segmentation of multi-spectral satellite images [182], characterization of the grain structure of nuclear fuel pellets 4], study of angiogenesis [19, segmentation of granular materials in 3D microtomography [10|11], and detection of the optic disc in fundus images [16. However, the computational cost of the Monte-Carlo simulation that estimates the PDF is a barrier to more wide-spread use. In this view, the prospect of efficiently computing the exact stochastic watershed PDF, without resorting to Monte Carlo simulation, is appealing. An important step in this direction was taken by Malmberg and Luengo [13, who proposed a pseudolinear algorithm for computing the exact stochastic watershed PDF for all edges of a tree.

Here, we have extended the work of Malmberg and Luengo by presenting a method for calculating the exact stochastic watershed PDF for all edges in a graph, given that the PDF is known for all edges included in the minimum spanning tree of the graph. Additionally we have presented a method that, via the concept of a boundary operator, transfers the PDF from the edges to the vertices of a graph. This allows exact stochastic watersheds to be computed on the pixels of an image, rather than on the abstract tree representation used in previous exact evaluation methods [15/13. We believe this makes the exact evaluation approach more attractive for use in practical applications. For sparse graphs, typical in image processing applications, the proposed method terminates in $\mathcal{O}(|V|)$ time, which is asymptotically smaller than the $\mathcal{O}(|E| \alpha(|V|))$ time required for calculating the PDF over the edges of the minimum spanning tree of the graph. Our experiments demonstrate that in practice, the computational cost of extending the PDF to all edges in a graph is small compared to the cost of computing the PDF on the edges of the MST of the graph.

In the original paper by Angulo and Jeulin [1, the PDF obtained by MonteCarlo simulation was convolved with a Gaussian function to obtain a smooth estimate of the true PDF. An interesting direction for future research is to incorporate this kind of relaxation in the proposed method by allowing larger boundary operators, i.e. not constraining the boundary operator to be a subset of the neighborhood of a vertex, and assigning appropriate weights to the elements of the boundary operator.

\section{References}

1. Angulo, J., Jeulin, D.: Stochastic watershed segmentation. In: Intern. Symp. on Mathematical Morphology, vol. 8, pp. 265-276 (2007)

2. Angulo, J., Velasco-Forero, S.: Semi-supervised hyperspectral image segmentation using regionalized stochastic watershed. In: Proceedings of SPIE Symposium on Defense, Security, and Sensing: Algorithms and Technologies for Multispectral, Hyperspectral, and Ultraspectral Imagery XVI. Proceedings of SPIE, vol. 7695, p. 76951F. SPIE, Bellingham (2010) 
3. Bender, M.A., Farach-Colton, M.: The LCA problem revisited. In: Gonnet, G.H., Viola, A. (eds.) LATIN 2000. LNCS, vol. 1776, pp. 88-94. Springer, Heidelberg (2000)

4. Cativa Tolosa, S., Blacher, S., Denis, A., Marajofsky, A., Pirard, J.P., Gommes, C.J.: Two methods of random seed generation to avoid over-segmentation with stochastic watershed: application to nuclear fuel micrographs. Journal of Microscopy 236(1), 79-86 (2009)

5. Cormen, T.H., Leiserson, C.E., Rivest, R.L., Stein, C.: Introduction to algorithms. MIT Press (2001)

6. Cousty, J., Bertrand, G., Najman, L., Couprie, M.: Watershed cuts: Minimum spanning forests and the drop of water principle. IEEE Transactions on Pattern Analysis and Machine Intelligence 31(8), 1362-1374 (2009)

7. Cousty, J., Bertrand, G., Najman, L., Couprie, M.: Watershed cuts: Thinnings, shortest path forests, and topological watersheds. IEEE Transactions on Pattern Analysis and Machine Intelligence 32(5), 925-939 (2010)

8. Cousty, J., Najman, L., Perret, B.: Constructive links between some morphological hierarchies on edge-weighted graphs. In: Hendriks, C.L.L., Borgefors, G., Strand, R. (eds.) ISMM 2013. LNCS, vol. 7883, pp. 86-97. Springer, Heidelberg (2013)

9. Cousty, J., Najman, L.: Incremental algorithm for hierarchical minimum spanning forests and saliency of watershed cuts. In: Soille, P., Pesaresi, M., Ouzounis, G.K. (eds.) ISMM 2011. LNCS, vol. 6671, pp. 272-283. Springer, Heidelberg (2011)

10. Faessel, M., Jeulin, D.: Segmentation of 3D microtomographic images of granular materials with the stochastic watershed. Journal of Microscopy 239(1), 17-31 (2010)

11. Gillibert, L., Jeulin, D.: Stochastic multiscale segmentation constrained by image content. In: Soille, P., Pesaresi, M., Ouzounis, G.K. (eds.) ISMM 2011. LNCS, vol. 6671, pp. 132-142. Springer, Heidelberg (2011)

12. Harel, D., Tarjan, R.E.: Fast algorithms for finding nearest common ancestors. SIAM Journal on Computing 13(2), 338-355 (1984)

13. Malmberg, F., Hendriks, C.L.L.: An efficient algorithm for exact evaluation of stochastic watersheds. Pattern Recognition Letters (2014)

14. Meyer, F., Beucher, S.: Morphological segmentation. Journal of Visual Communication and Image Representation 1(1), 21-46 (1990)

15. Meyer, F., Stawiaski, J.: A stochastic evaluation of the contour strength. In: Goesele, M., Roth, S., Kuijper, A., Schiele, B., Schindler, K. (eds.) DAGM 2010. LNCS, vol. 6376, pp. 513-522. Springer, Heidelberg (2010)

16. Morales, S., Naranjo, V., Angulo, J., Alcaniz, M.: Automatic detection of optic disc based on PCA and mathematical morphology. IEEE Transactions on Medical Imaging 32(4), 786-796 (2013)

17. Najman, L., Schmitt, M.: Geodesic saliency of watershed contours and hierarchical segmentation. IEEE Transactions on Pattern Analysis and Machine Intelligence 18(12), 1163-1173 (1996)

18. Noyel, G., Angulo, J., Jeulin, D.: Classification-driven stochastic watershed: application to multispectral segmentation. In: IS\&T's Fourth European Conference on Color in Graphics Imaging, and Vision (CGIV 2008), pp. 471-476 (2008)

19. Noyel, G., Angulo, J., Jeulin, D.: Regionalized random germs by a classification for probabilistic watershed application: Angiogenesis imaging segmentation. In: Fitt, A.D., Norbury, J., Ockendon, H., Wilson, E. (eds.) Progress in Industrial Mathematics at ECMI 2008. Mathematics in Industry, pp. 211-216. Springer (2010) 